\title{
Prove of hydrogen formation through direct potential measurements in the rolling slit during cold rolling
}

\author{
S.V. Merzlikin ${ }^{1,2}$, M. Wildau ${ }^{3, \star}$, K. Steinhoff ${ }^{4}$ and A.W. Hassel ${ }^{2}$ \\ 1 Max Planck Institute for Iron Research, Max-Planck-Str. 1, 40237 Düsseldorf, Germany \\ 2 Institute for Chemical Technology of Inorganic Materials, Johannes Kepler University Linz, \\ Altenberger Str. 69, 4040 Linz, Austria \\ e-mail: achimwalter.hassel@jku.at \\ 3 Ingenieurbüro Dr.-Ing Monika Wildau, Am Kerper Weiher, 41352 Korschenbroich, Germany \\ 4 Steinhoff Kaltwalzen GmbH, Gerhard-Malina-Str. 65, Dinslaken, Germany
}

\section{Key words:}

Cold rolling; forged rolls; hydrogen embrittlement; hydrogen; open circuit potential; friction; tribo-corrosion; hydrogen wear
Received 28 August 2013

Accepted 23 January 2014

\begin{abstract}
In this work, direct potential measurements during cold rolling of zinc and $\mathrm{X} 20 \mathrm{Cr} 13$ stainless steel were carried out in the rolling slit to follow the tribologic and galvanic mechanisms of hydrogen formation and absorption on the surface of the working rolls made of $\mathrm{DHQ} 1$ grade steel. $\mathrm{An} \mathrm{Ag} / \mathrm{AgCl}$ in $3.5 \mathrm{M} \mathrm{KCl}$ reference microelectrode was used to record the open circuit potential of the electrochemical system roller-product immersed into commercially relevant electrolyte (rolling emulsion) with a $\mathrm{pH}$ value of 4.5 and an electric conductivity $46 \mathrm{mS} \mathrm{cm}^{-1}$. The potential shift into either negative or positive direction of the rolls-product system gives information on the processes taking place at the surface in the course of the friction. A detailed discussion of the in-situ potentiometry experiments reveals a stationary situation established between the destruction and repassivation of the surface structures during continuous cold rolling accompanied with intensive hydrogen evolution. Galvanic coupling of the working rolls with the product significantly intensifies the hydrogen embrittlement related problems of the rolls. Atomic hydrogen is adsorbed on the surface and exhibits a pressure supported absorption into the rolls during their whole lifetime.
\end{abstract}

old rolling is an essential technological step used in the steel industry to manufacture sheets, strips and foils with extremely smooth surfaces and accurately controlled dimensions. Continuous rotation at high velocities and loads makes a mechanical failure of a forged roll very dangerous for employees and equipment of the plant. Among the possible damage reasons are irregularities of the roll, such as residual stresses due to inclusions or segregations, which may lead to local heating or local mechanical overloads during the rolling process. Recently a number of mechanical failures of the rolls during rolling attributed to hydrogen embrittlement (HE) were reported by the steel industry. It is well known from industrial applications such as fuel pipelines, parts of the machines in aggressive environment, hydrogen storages,

\footnotetext{
$\star$ Deceased on 23/12/2013.
}

power-plant boiler pipes etc. that steels suffer from HE. In fact, as it was pointed out by Bernstein [1], hydrogen embrittlement is a severe environmental type of failure that affects not only steels but almost all metals and alloys. Experiments of Nagumo [2] showed that high strength steels in particular are highly susceptible to hydrogen embrittlement. Under certain conditions hydrogen exists in statu nascendi on the metal surface when it was just formed from water or other hydrogen containing compounds. Before this atomic hydrogen combines to molecular hydrogen, which can be released into the adjacent atmosphere, it may be absorbed by the metal. Hydrogen is inimitable among all atoms, since its ionised form, the proton has no further electron and thus the diameter decreases dramatically to that of a nucleus (in case of deuterium) or that of an elementary particle. It can diffuse in 
the metal and at particular places it can be trapped inside the material as it was shown by Hirth [3]. In presence of hydrogen steels fail at far lower load levels compared with those that steel free of hydrogen can sustain. Up to now there is no single concept of $\mathrm{HE}$ accepted because all of the observations cannot be accounted for by a single mechanism. There is a very informative review of Sofronis and Robertson [4] summarizing different mechanisms of HE. The same behaviour considering the degradation of the mechanical properties was observed in our previous works $[5,6]$ for hardened forged rolls for cold rolling, where molecular hydrogen accumulated at some points in the near-surface region of the rolls resulting in heavy spalling and blistering of the material. Numerous characteristic $0.5-1 \mathrm{~mm}$ long cracks formed on the working surface of the roll in the vicinity of blisters, as well as subsurface ( 2 to $8 \mathrm{~mm}$ below the surface) fatigue cracks point at the hydrogen induction of this mechanical failures.

The origin of hydrogen and the driving force of hydrogen formation during cold rolling are of great practical and scientific interest. The mixed open circuit potential of the roll-product system during the cold rolling, along with the tribochemical aspects, was found to be one of the driving forces for hydrogen evolution by Shpenkov [7].

During cold rolling enormous friction between metals in a metallic couple rollproduct stripe immersed into rolling emulsion allows building up an electrochemical potential difference. Two principle mechanisms are imaginable. Rolling a less noble metal such as zinc or zinc coated steel with a steel roll generates conditions in which the two metals are on one hand in good electrical contact with each other and on the other hand are both immersed in the same electrolyte which in this case is the rolling emulsion. This mechanism is further referred to as galvanic mechanism. The second mechanism is the tribochemical mechanism in which the high mechanical stress causes rupture of the passive film which may chemically repassivate. Akiyama et al. [8], Abelev et al. [9], Hassel and Smith [10] found that such a repassivation is going via splitting of water from the emulsion.
Table 1. Chemical composition of the DHQ 1 steel.

\begin{tabular}{ccccc}
\hline \multicolumn{5}{c}{ Elemental concentration, wt.\% } \\
\hline Steel grade & $\mathrm{C}$ & $\mathrm{Si}$ & $\mathrm{Cr}$ & $\mathrm{Mo}$ \\
\hline DHQ1 & 0.80 & 0.75 & 2.10 & 0.34 \\
\hline
\end{tabular}

The stress or pressure applied between a roll and a strip plays a great role in the tribomechanical mechanism. Weisz-Patrault et al. [11] showed the evaluated normal gap pressure during the cold rolling to be in the order of $1500 \mathrm{MPa}$ with a maximum at the point of roll-product stripe contact, which is the interface of the tribogalvanic element where all electrochemical and tribochemical processes take place. One metal becomes a cathode and another acts as anode being destroyed by the mechanism of electrochemical corrosion. Galvanic coupling of different rolls and metals in the product strip are also playing an important role. The processes of corrosion and hydrogen formation attributed to it can be investigated by measuring the difference between the electrode potentials of this metallic couple.

The novel approach presented in this work is to make use of these aspects and to measure an indication of hydrogen absorption and hydrogen-related mechanical failure of the rolls through a direct measurement of the electrochemical potential in the rolling slit during rolling.

\section{Experimental}

A pair of forged model rolls with diameter of $64 \mathrm{~mm}$ were specially designed and made from DHQ 1 steel (Tab. 1, Steinhoff Kaltwalzen) to fit the manual rolling mill (Type K65, DIMA Maschinen GmbH), Figure 1.

One pair of the rolls made of $42 \mathrm{CrMo} 4$ steel was hard chrome plated resulting in a 70-100 $\mu \mathrm{m}$ thick hard chrome layer. Commercial rolling oil KT5.3 (Quackeroel) was used and 2 wt.\% of the oil was mixed with deionized water to prepare the rolling emulsion. The electric conductivity and $\mathrm{pH}$ value of the emulsion were measured to be $46 \mathrm{mS} \mathrm{cm}^{-1}$ and 4.5 (at $22{ }^{\circ} \mathrm{C}$ ) correspondingly. Fresh emulsion has been continuously stirred and added in the beginning of each individual rolling experiment, the stability 


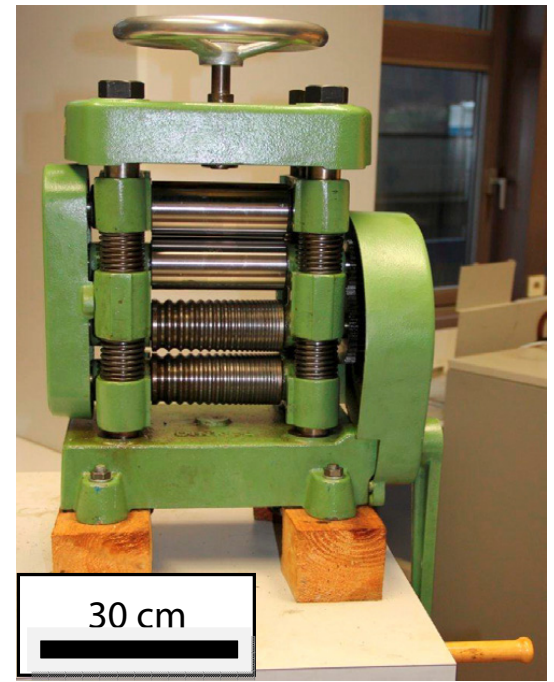

Fig. 1. Manually powered rolling mill.

of the electrolyte was visually controlled. $100 \mathrm{~mm}$ wide zinc foil and X20Cr13 stainless steel strips were used as rolling stock. Rolling speed was $0.06 \mathrm{~m} / \mathrm{s}$ (or $18 \mathrm{rpm}$ ), front and back tensions were equal to zero, strip thickness at entry was $1.2 \mathrm{~mm}$, at exit approx. $1.1 \mathrm{~mm}$. Since no direct measurement of contact pressure or load was available on the mill used, the roll separation force has been kept constant mechanically through the whole series of experiments by identical setting of the screwdown mechanism. Potentials were measured directly by using a $\mathrm{Ag} / \mathrm{AgCl}$ (3.5 M KCl, $205 \mathrm{mV}$ vs. Standard Hydrogen Electrode (SHE)) micro electrode with solid electrolyte on agar basis, developed by Hassel et al. [12], inside a $1 \mathrm{~mm}$ thick glass capillary as reference electrode. Signal conditioning, data acquisition and recording was performed through a potentiostat (SimPot, HHU Düsseldorf, Germany) directly in the gap between the rolls during rolling of the material (Fig. 2).

Here, the rolls act as working electrode (WE) and the micro-reference electrode (RE) was immersed into the electrolyte (emulsion). Since only potentiometry was performed a counter electrode was not necessary. Initial experiments were performed with an electrical steel rolling mill (Type K120/160, DIMA Maschinen GmbH) in which rolls from the same material, also prepared specifically for these experiments, were used. These experiments showed a very high noise level due to the unavoidable strong vibrations generated during rolling with the electric mill. Furthermore, the precise positioning of the reference electrode was not guaranteed because of these vibrations. It therefore sometimes led to the destruction of the RE. In case of the manual rolling mill a stable fixation of the RE and a constant distance between the RE and WE provided reliable in-situ potential measurements.

Determination of the total hydrogen concentration of the steel samples was obtained through melt extraction method, using an ELTRA OH 900 hydrogen analyzer (ELTRA $\mathrm{GmbH}$, Neuss, Germany). Calibration of the analyzer was carried out using helium gas and reference material (LECO Corporation, St. Joseph, MI, USA) with certified hydrogen concentration. Prior to analysis the samples were cleaned with acetone, rinsed with ultrapure deionised water (PURELAB Pulse Water Purification System, ELGA Lab Water, Marlow, UK) and finally cleaned with methanol and $\mathrm{CCl}_{4}$ (p. a., Sigma Aldrich).

\section{Results and discussion}

\subsection{Potentiometric monitoring of the rolling process}

First, the chrome-plated pair of rolls was mounted onto the rolling mill. A zinc foil and a stripe made of stainless steel were processed using 2\% KT5.3 emulsion. The change of the open circuit potential during rolling is shown in Figure 3. After product material was fed into the rolls and wetted with the electrolyte, the electrode potential of the metallic couple gradually shifted to its stable value. After a steady potential was established, the rolls have been moved (this moment marked with a red arrow in Fig. 3). Regardless of the product being rolled, the potential changed rapidly in the direction of negative values. A sharp change of the potential at the beginning of rolling interaction is due to the friction-induced destruction of the topmost oxide layer and deformational activation of the metallic surfaces. The open circuit potential of the system then restored its initial value if given enough relaxation 


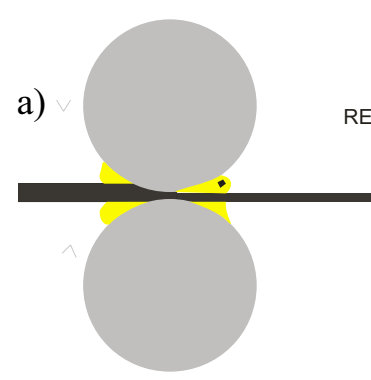

WE

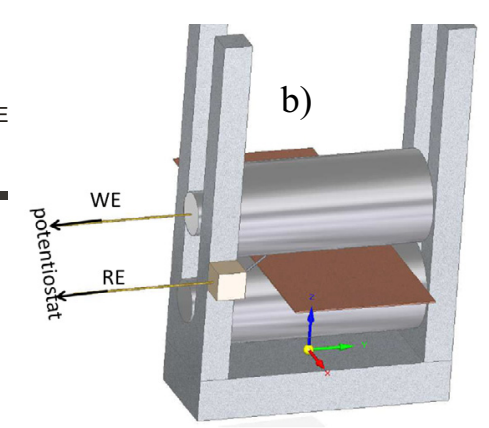

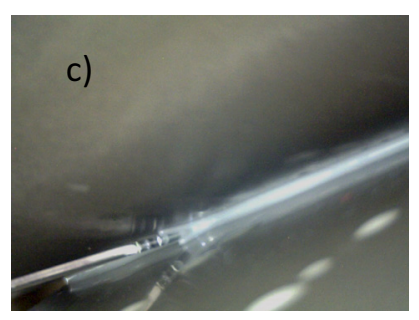

Fig. 2. Scheme $(a, b)$ and (c) photo of the in-situ potentiometry experiment.
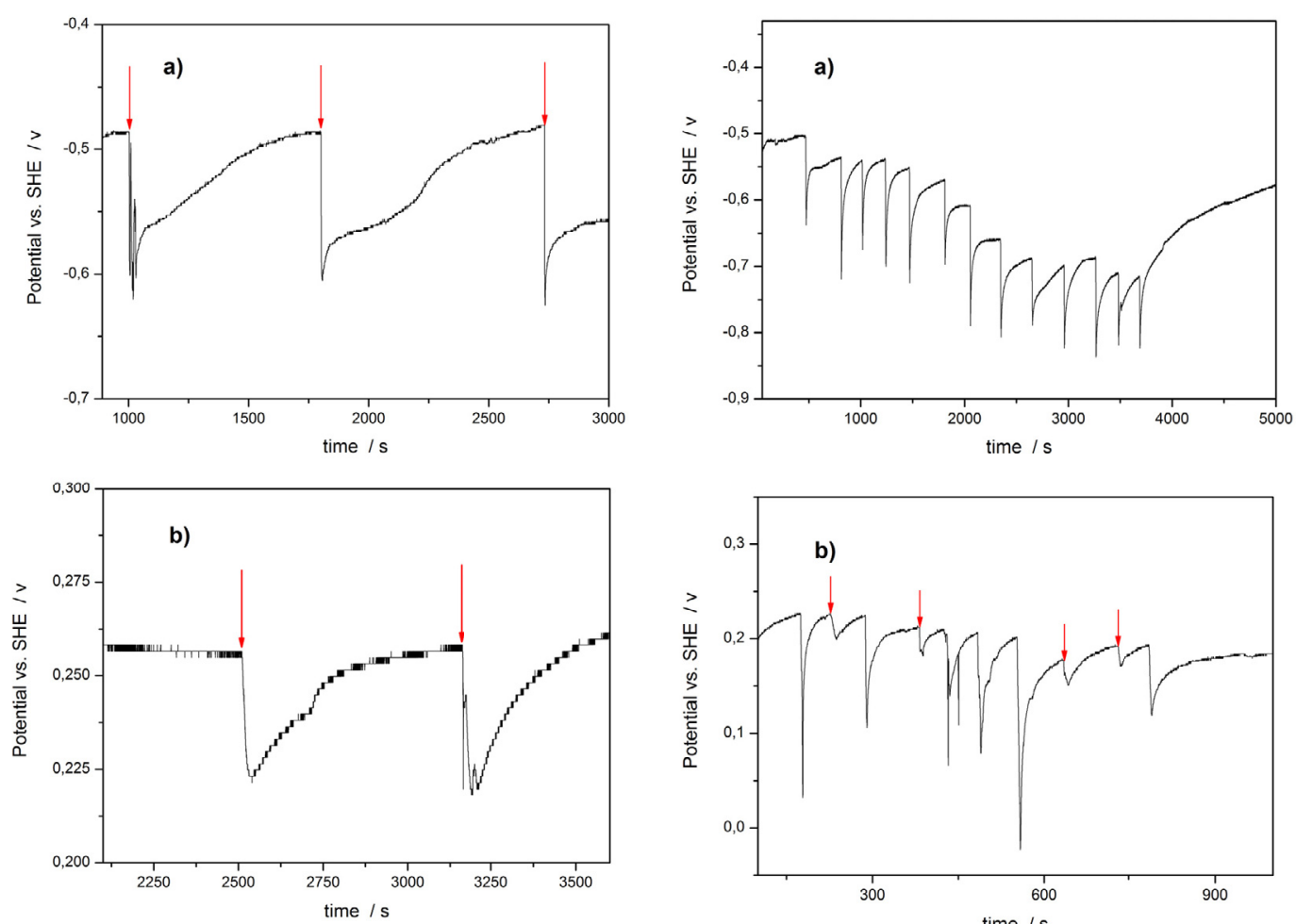

Fig. 3. Electrode potential (vs. SHE) during controlled cold rolling of (a) zinc foil, (b) stainless steel; working rolls - chrome-plated $42 \mathrm{CrMo}$.

time (some tens of minutes). This effect is typically observed for passivating materials as it was demonstrated by Celis et al. [13] and Mischler et al. [14] in a series of tribocorrosion experiments. The restoration of the open circuit potential is also an indirect confirmation of the emulsion stability during the rolling experiment.

If there is insufficient time for the system to relax, the open circuit potential stabilizes

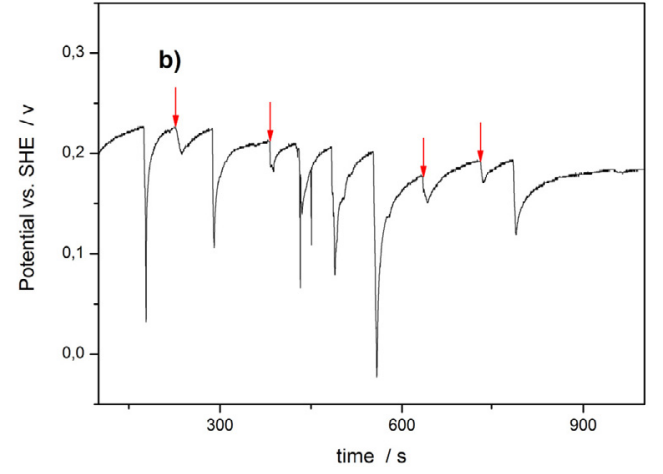

Fig. 4. Electrode potential (vs. SHE) during the short cold rolling pulses of: (a) zinc foil, (b) stainless steel; working rolls - chrome-plated 42 CrMo4.

at lower values as for example shown in Figure 4 for short continuous pulses of cold rolling. This is a proof of a metastable state of the surface under friction. The potential was then restored at a value somewhat lower than the initial one as a layer of native oxide was formed on the surface of both rolls and the product. 

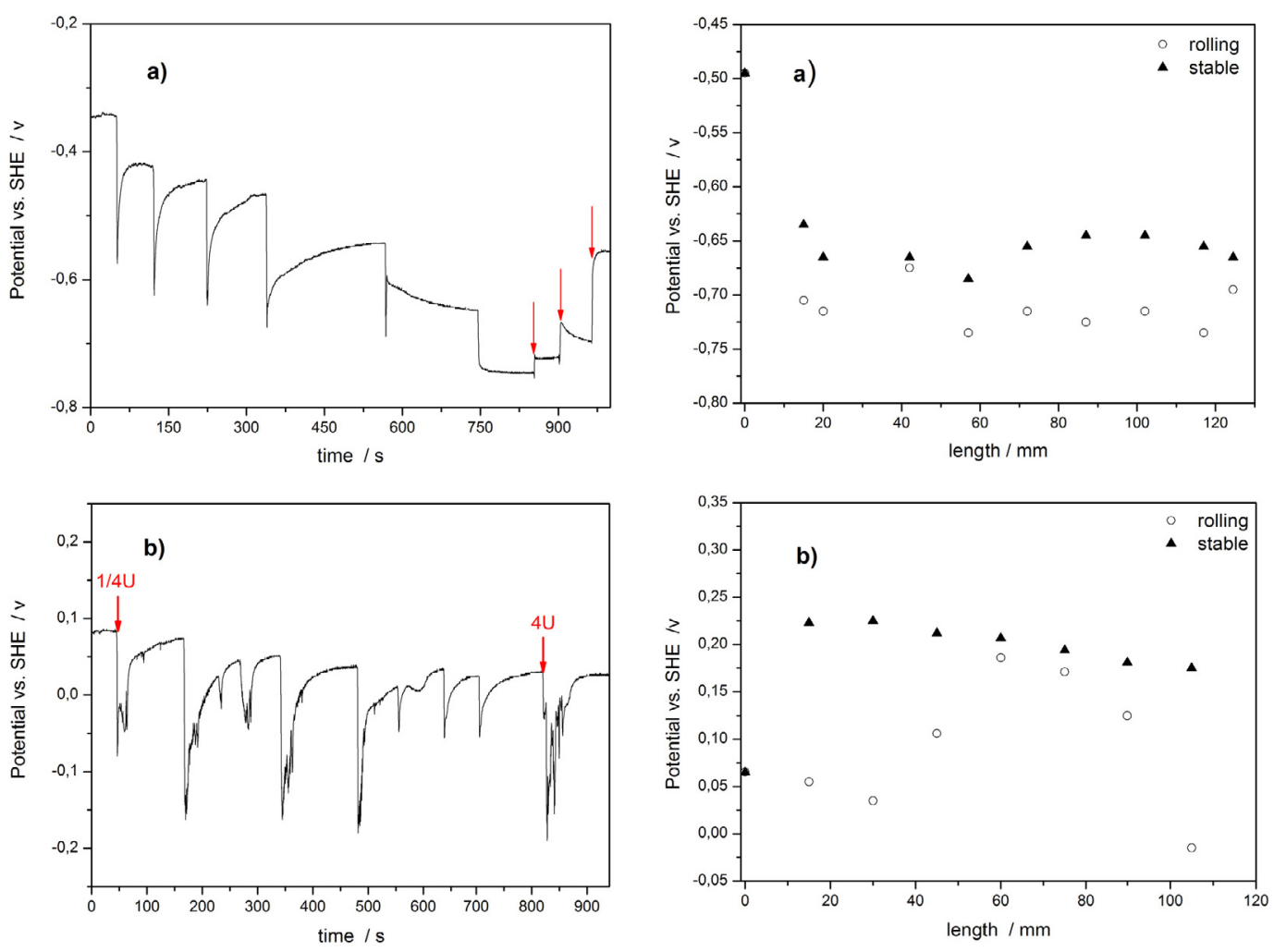

Fig. 5. Electrode potential (vs. SHE) during the short cold rolling pulses of: (a) zinc foil, (b) stainless steel; working rolls - DHQ 1 steel.

Fig. 6. Electrode potential (vs. SHE) as a function of length of the product's initial position displacement during rolling: (a) zinc foil, (b) stainless steel; working rolls - chromeplated 42CrMo4.

The whole contact area of the electrodes during the rolling is in the metastable state in which an electrode potential depends on the velocity of rolling, as reported by Shpenkov [7] and more recently, Rossi et al. [15] claimed also the dependence of the potential on contact pressure. Celis et al. [13] found out that even the direction of the rotation influences electrode potential. This phenomenon was also seen during the investigation in Fig. $4 b$ ), where a lower rotation speed of the rolls is marked with red arrows. The data can be found in the literature, that lower contact pressure in such tribosystem results in higher changes of the open circuit potential, as for example in the work of Shyrokov and Vasyliv [16]. In present case, the contact pressure was the same for each measurement as a very long stripe of the product was used without readjusting the setup.

In Figure 5 the results for the pair of rolls made of DHQ1 steel are shown. Red arrows in Figure 5a indicate rolling in the

same direction at constant velocity, but with the opposite change in the electrode potential of the system. The direction of potential change is opposite for the same working rolls at the same rolling direction, force, load and speed. In order to explain such a behavior, the potential during rolling and the one immediately after rolling (when a steady state was established) was plotted against the distance against initial position of the product, that is its length (Figs. 6 and 7). The total circumference of the roll's surface was $200 \mathrm{~mm}$. An initial steady state potential of $-0.49 \mathrm{~V}$ to $-0.35 \mathrm{~V}$ (zinc foil) and $-0.07 \mathrm{~V}$ to $-0.09 \mathrm{~V}$ (stainless steel) vs. SHE has settled at a level of mixed potential of two metals (at the given conditions, according to the reference values of the standard potentials in aqueous solutions taken from the Milazzo et al. [17], Bard et al. [18] and Bratsch [19]) at zero length. During cold rolling, the potential was decreasing to about 

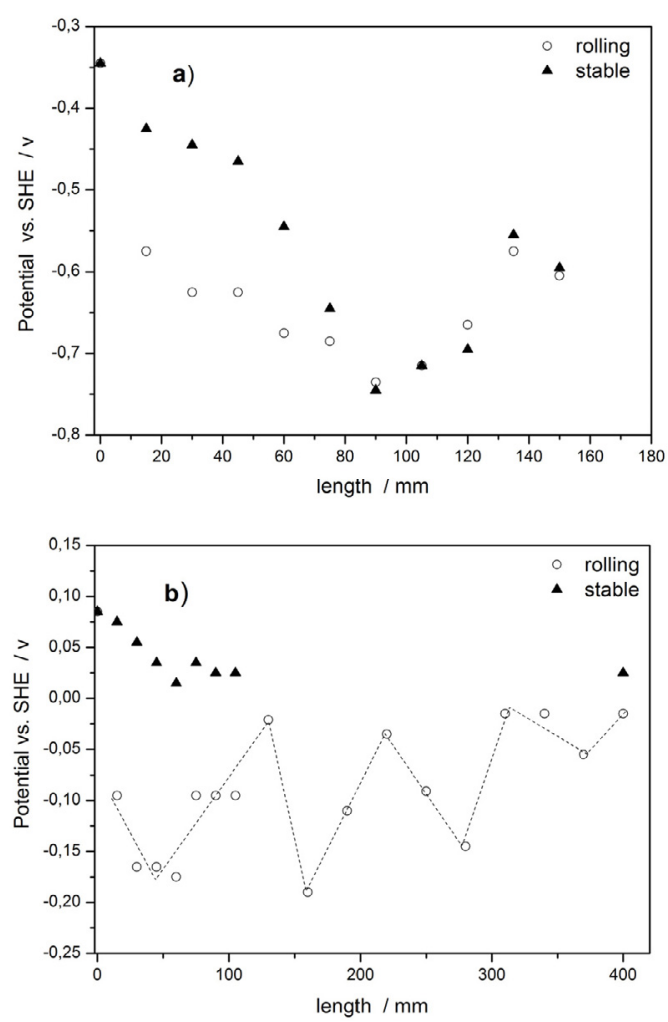

Fig. 7. Electrode potential (vs. SHE) as a function of length of the product's initial position displacement during rolling: (a) zinc foil, (b) stainless steel; working rolls - DHQ 1 steel.

a half of the roll's circumference, afterwards increasing. The effect was more pronounced in the case of non-plated steel rolls (Fig. 7). Provided sufficient time for relaxation, the stable state potential tends to restore its original value. If one takes a look at the longer measurement in Figure $7 \mathrm{~b}$, where potential's changes along two full rounds of rolling are shown, a quasi-periodic oscillation of the open circuit potential with respect to an imaginary zero-length point on the roll can be seen (dashed line). This is the reflection of a dynamic equilibrium between the destruction and recovery of the oxide layer and a mass transport of the wear particles originating from the metals in the electrolyte, this fact being in a good correspondence with the analysis of the electrochemically controlled tribocorrosion experiment by Landolt et al. [20], who took into account the formation, flux and ejection of these wear particles from the sliding contact. The potential shifts towards negative values because of the destruction of the oxide surface layer of metal were observed also in the review on electrochemical methods in tribological systems by Landolt et al. [21]. The same behaviour of the potential was observed by Oltra et al. [22], who followed an evolution of the corrosion potential from the passive range towards the active range during continuous abrasion of the steel sample in diluted sulphuric acid. This destruction phase is followed by the phase of passivation of the surface. The phase of repassivation of the bare steel surface submerged into the electrolyte manifests itself in a rise of the electrode potential. These phases replace each other cyclically, following the rotation of the rolls and thus causing the observed sharp oscillations of the electrode potential. The same observation was found by Sholud'ko [23] for different electrolytes. According to his results the potential change depends to a large degree on the material of the friction parts and on the presence of oxidizing/reducing agents in the electrolyte.

\subsection{Hydrogen evolution during cold rolling}

\subsubsection{Thermodynamics}

The changes in the potential are generally due to the changes in the energy state of the surface during and after friction. In order to estimate a thermodynamic probability of any phenomenon on the surface of metals in aqueous electrolyte solutions, Pourbaix diagrams [24] can be used. The lines for the regions of stability, corrosion and passivation of metals in Pourbaix diagrams ( $E$ vs. $p H$ ) obey under equilibrium conditions the Nernst equation:

$$
E=E^{0}-\frac{R T}{z F} \ln \left(\frac{a_{\mathrm{Red}}}{a_{\mathrm{Ox}}}\right)
$$

where $E$ is the equilibrium potential at given temperature, $E^{0}$ is the standard potential, $F$ is the Faraday constant, $R$ is the universal gas constant, $T$ is the absolute temperature; $z$ is the number of moles of electrons; $a_{\text {Red }}$ and $a_{\mathrm{Ox}}$ are the chemical activities of the reductant and oxidant respectively. Since the activity factor tends to unity at low concentrations, activities in the Nernst equation can 


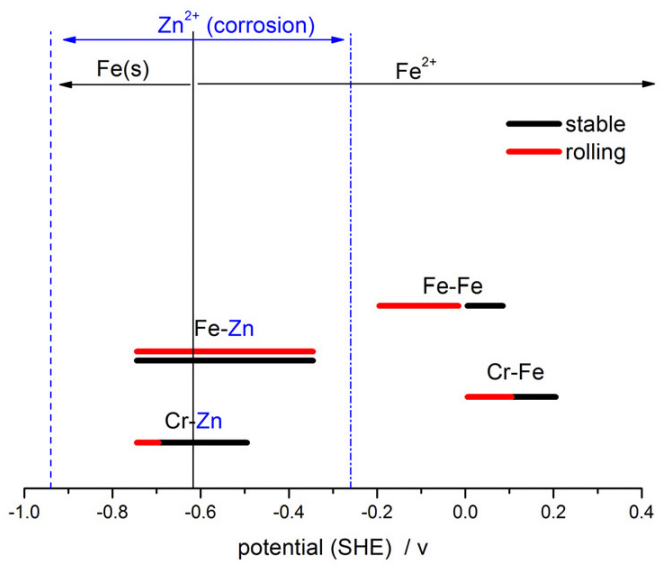

Fig. 8. Measured intervals of the OCP change in the system rolls-product.

be simply considered as concentrations of the components which are being reduced or oxidized during the reaction. During cold rolling, metals are being exposed to severe friction. As a result, Gibbs thermodynamic potential of the metal changes on value $\Delta G$, respectively changing the equilibrium potential E:

$$
\Delta E=-\frac{\Delta G}{z F} .
$$

This fact in its turn leads to the shift of the lines and regions of the Pourbaix diagrams. One can therefore introduce the specific Pourbaix diagram of the system under friction (e.g. cold rolling) as proposed by Celis et al. [13]. Dedy et al. [25] in his work made some attempts to create the friction maps in conjunction with the equilibrium Pourbaix diagram for the copper-water system. But unfortunately, there is no detailed information or atlases of Pourbaix diagrams for the vast majority of such tribo-systems. The thermodynamic state of the metal under non stationary conditions, including the changes in electrode potential can be still evaluated by the classical equilibrium Pourbaix diagrams. Pourbaix diagrams for $\mathrm{Fe}-$ $\mathrm{H}_{2} \mathrm{O}, \mathrm{Zn}-\mathrm{H}_{2} \mathrm{O}$, and $\mathrm{Cr}-\mathrm{H}_{2} \mathrm{O}$, systems were considered for the surfaces contacted under friction during the cold-rolling process.

From the diagrams for $\mathrm{Fe}$ and $\mathrm{Zn}$ in aqueous solutions with $p H=4.5$, at the values of electrode potential measured during the cold rolling (see Fig. 8) the dissolution of bare metal can occur through the following anodic reaction, $\mathrm{Me} \rightarrow \mathrm{Me}^{2+}+2 \mathrm{e}^{-}$, where Me is
Fe or Zn. It is accompanied with the cathodic reaction $\mathrm{H}_{2} \mathrm{O}+\mathrm{e}^{-} \rightarrow \mathrm{H}_{\mathrm{ad}}+\mathrm{OH}^{-}$, where $\mathrm{H}_{\mathrm{ad}}$ is a hydrogen atomically adsorbed to the surface of the metal. Anderson et al. [26] studied the behaviour of surface potentials of different metallic electrodes, scraped inside the solutions with different $\mathrm{pH}$ and described the phenomenon of an accelerated hydrogen evolution on freshly cleaved metallic surfaces in aqueous medium; it has been widely reported since then in the literature. For example, Burstein and Kearns [27] did an excellent study on describing an accelerated evolution of hydrogen from freshly generated surfaces of $\mathrm{Cr}$, Fe and their alloys.

From the thermodynamic analysis of chromium the mechanisms of anodic dissolution and passivation were elaborated by Tsuru [28], Bjornkvist and Olefjord [29] did it for acidic solutions. The experiments on the system $\mathrm{Cr}-\mathrm{H}_{2} \mathrm{O}$ to establish the products of chromium dissolution were made also by Drazic and Popic [30]. From their results it is clear that a layer of $\mathrm{Cr}(\mathrm{OH})_{2}$ is formed on chromium surface at the potentials of $\mathrm{Cr}-\mathrm{Fe}$ couple from Figure 8. The mechanism is the following:

$$
\begin{aligned}
& \mathrm{Cr}+\mathrm{H}_{2} \mathrm{O} \leftrightarrow \mathrm{CrOH}_{\mathrm{ad}}+\mathrm{H}^{+}+\mathrm{e}^{-} \\
& \mathrm{CrOH}_{\mathrm{ad}}+\mathrm{H}_{2} \mathrm{O} \rightarrow \mathrm{Cr}(\mathrm{OH})_{2, \mathrm{ad}}+\mathrm{H}^{+}+\mathrm{e}^{-} \\
& \mathrm{Cr}(\mathrm{OH})_{2, \mathrm{ad}}+2 \mathrm{H}^{+} \leftrightarrow \mathrm{Cr}^{2+}(\mathrm{aq})+2 \mathrm{H}_{2} \mathrm{O} \\
& \text { in case of dissolution } \\
& \mathrm{Cr}(\mathrm{OH})_{2, \mathrm{ad}} \leftrightarrow \mathrm{CrOOH}_{\mathrm{ad}}+\mathrm{H}^{+}+\mathrm{e}^{-} \\
& \text {in case of passivation. }
\end{aligned}
$$

In the region of the Pourbaix diagram, which is corresponding to the $\mathrm{Cr}-\mathrm{Zn}$ couple during the cold rolling experiment, the final step is the passivation.

As it has been shown above, with the initiation of the rolling process the surface oxide layer is being destroyed and a fresh metallic surface is exposed to the electrolyte at the side of RE (Fig. 9). In the course of further rolling this film is continuously destroyed again and again. After a while, due to the self-organization of the destroyed friction surface, the passive film is restored in the interaction between the activated friction surface and the electrolyte. These processes are inevitable during the cold rolling. As shown by Anderson et al. [26] and Burstein and Kearns [27] they 


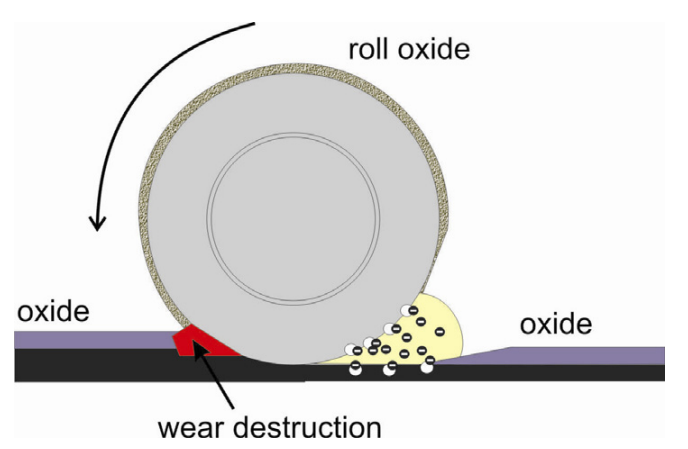

Fig. 9. Scheme of friction surface of metals during the cold rolling.

are always accompanied by the formation of hydrogen on the freshly exposed metallic surface in the presence of aqueous media containing hydrogen ions.

Moreover, as it can be seen in Figures 3 and 4 for chrome plated working rolls, as well as in Figures 5 and 7 for DHQ-1 rolls, the sign of the potential during zinc and stainless steel rolling is opposite: negative for zinc and positive for stainless steel. This difference in potentials can also impact the process of hydrogen ingress into the working roll. Hydrogen diffuses into the bulk of the material from the surface because of the concentration gradient, since there is more hydrogen on the surface, where hydrogen is being formed, adsorbed and absorbed. The sign of the potential can only play a role in accelerating the proton adsorption on the surface of the electrode. If the open potential of the metal is more negative than the electrode potential at point of zero charge (pzc), than the surface is negatively charged and attracts cations. Thus, hydrogen atoms will be readily adsorbed on it. For iron $E_{\mathrm{pzc}}$ is equal to $-0.35 \mathrm{~V}$ (SHE) [31], being clearly less negative than values of the potential in Figure 7a for Fe-Zn couple in steady state and during friction $(-0.36$ to $-0.75 \mathrm{~V}$ (SHE) accordingly), favoring $\mathrm{H}_{\mathrm{ad}}^{+}$adsorption on the roll. In case of stainless steel strip (Fe-Fe situation in Fig. $7 \mathrm{~b}$ ), observed potential (which was $0.09 \mathrm{~V}$ in steady state and became negative down to $-0.2 \mathrm{~V}$ during rolling) were more positive than $E_{\mathrm{pzc}}$ for iron, showing that (thermodynamically) the surface of the electrode has to be charged positively, i.e. proton repulsive. But during the friction the open potential of the system tends to go deeper in the negative direction towards the scribe potential of the Fe (-0.44 V SHE), where the surface again becomes negatively charged and proton attractive. Moreover, in real situation, where fresh and passivated metal (iron) surface immersed simultaneously into one electrolyte, a passivated iron surface is an anode, whereas fresh surface is a cathode where hydrogen evolution takes place.

\subsubsection{Galvanic coupling}

While in the case when stainless steel is coldrolled with working rolls made of DHQ 1, hydrogen evolution appears mainly because of the fresh formed metallic surfaces exposed to the emulsion, other roll-product combinations have their own peculiarities. When the rolls-product system is built up from different metals, galvanic coupling contributes additionally to the changes in open circuit potential of the system and can lead to an enhanced hydrogen production. In the galvanic couple $\mathrm{Fe}-\mathrm{Cr}$, both metals are close to each other in standard potential series, thus developing only a very small potential difference. Hard chromium plating of the working rolls is known to have good corrosion resistance, thus successfully preventing hydrogen diffusion into the rolls. The surface of an average chromium plated roll was investigated by Simao and Aspinwall [32]. They found chromium deposit to have various microscopic cracks resulting in a network of open cracks on the surface of the coated layer. Fedrizzi et al. [33] claimed pitting-like corrosion morphology can be possibly initiated at these pre-existing cracks. However, the corrosion phenomena were found to be of secondary importance, because the material removal during the wear-corrosion occurred mainly through an adhesive mechanism. An important aspect of hard chromium coating of the rolls is however, the environmental risk associated with the hexavalent chromium used in the plating process, which is a carcinogen and has several serious concerns like long term legal liability for worker health and safety, waste disposal and soil contamination. Recent regulation efforts, including the OSHA $\mathrm{Cr}^{6+}$ PEL in the USA and the European RoHS, ELV and WEEE regulations 
directives, resulted in new tougher rules been introduced worldwide. These latest developments present a formidable array of restrictions for manufacturers and surface finishers, and the ban of the hexavalent chromium is to be expected in the near future. There are already working knowhows for hard chromium functional coatings using trivalent chromium electroplating [34]. Unlike for the hexavalent chromium plating, where cathodic DC current is being used, a modified waveform including cathodic and anodic DC current, followed by relaxation phase was used in this process. An equivalent to hexavalent chromium microstructure and hardness, decreased porosity and good adhesion at a $250 \mu \mathrm{m}$ thickness was obtained [35]. Since during electroplating $\mathrm{Cr}^{3+}$ is reduced at the cathode (surface of the roll) to metallic chromium by gaining three electrons, no change in the corrosion potential is expected. Therefore, mainly mechanical properties of the chromium hard coating, such as surface stability and hardness play an important role in the prevention of hydrogen diffusion into the roll, good performance of chromium-coated rolls with respect to HE will not suffer from replacement from $\mathrm{Cr}^{6+}$ to $\mathrm{Cr}^{3+}$ plating bathes. The most severe case of hydrogen evolution occurs in galvanic couples with zinc. The contact between these metals is so intimate that even the formability depends on the crystallographic texture as it was shown in the work by Signorelli et al. [36], and thus is strong enough to allow for strong galvanic coupling. The Fe- $\mathrm{Zn}$ couple at $p H=4.5$ (the case when zinc foil is cold rolled with normal, non-chrome plated rolls) the product $(\mathrm{Zn})$ acts as a sacrificial anode $\mathrm{Zn} \rightarrow \mathrm{Zn}^{2+}+2 \mathrm{e}^{-}$, since iron is more noble than zinc according to the electrochemical series (Milazzo et al. [17], Bard et al. [18] and Bratsch [19]). The evolution of hydrogen takes place at the cathode, which is the surface of the roll, according to the cathodic half reaction:

$$
\begin{aligned}
& 2 \mathrm{H}^{+}+2 \mathrm{e}^{-} \rightarrow \mathrm{H}_{2} \uparrow \\
& \mathrm{H}^{+}+\mathrm{e}^{-} \rightarrow \mathrm{H}_{\mathrm{ad}} \\
& \mathrm{O}_{2}+4 \mathrm{e}^{-}+4 \mathrm{H}^{+} \rightarrow 2 \mathrm{H}_{2} \mathrm{O} .
\end{aligned}
$$

Another critical point is the high overpotential of hydrogen evolution on zinc which successfully hinders hydrogen evolution and

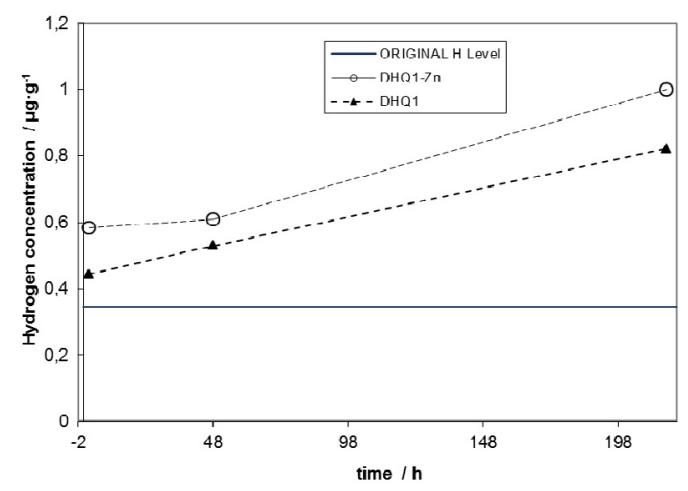

Fig. 10. Total hydrogen amount $\left( \pm 0,01 \mu \mathrm{g} \cdot \mathrm{g}^{-1}\right)$ of the samples made of DHQ 1 in $2 \%$ KT5.3 emulsion, with and without contact to zinc foil.

therefore redirects the hydrogen formation to the susceptible rolls.

The following simple experiment illustrates this behaviour. DHQ 1 steel samples in electric contact with a zinc foil were immersed into the $2 \%$ KT5.3 emulsion used for cold rolling. The change of the total hydrogen concentration of the steel samples was measured as a function of time spent in the solution. As it can be seen from Figure 10, the total hydrogen content of the steel samples connected to zinc rises significantly stronger than that of uncoupled samples.

Formed in this way nascent hydrogen is initially adsorbed on the surface of the rolls in its atomic rather than molecular form. The concentration gradient of hydrogen between bulk and the surface of the rolls delivers the driving force for the hydrogen diffusion, further supported by the enormous pressure during the cold rolling. In cases where the rolling emulsion contains surfactants the surface concentration of atomic hydrogen can be further increased by hindering the recombination of adsorbed atomic hydrogen to the molecule. The concentration of $\mathrm{H}_{\mathrm{ad}}$ on the surface of the rolls increases thus promoting its absorption. Deformation and high temperatures on sliding metallic surfaces increase atomic hydrogen's solubility and diffusion. Since the hydrogen formation is continuously happening during the cold-rolling, large amounts of hydrogen diffuse into the rolls increasing the risk of hydrogen-induced embrittlement. 
Main stages of hydrogen-induced mechanic failure of the working rolls can be formulated:

Hydrogen evolution from hydrogenous lubricants due to the tribochemical reactions and high surface temperature in the friction zone + hydrogen evolution from the freshly formed surfaces of the friction zone submerged in the aqueous rolling emulsion $\rightarrow$ accompanied with the adsorption of atomic hydrogen on the surface of the rolls $\rightarrow$ followed by diffusion of the atomic hydrogen into the rolls supported by the concentration, temperature and stress gradients from the surface to the bulk $\rightarrow$ absorption and subsequent recombination of the atomic hydrogen inside the defects of the surface stressed layer $\rightarrow$ resulting in a brittle mechanic failure as a result of formation and coalescence of the individual subsurficial cracks.

\section{Conclusions}

Through in situ measurement of the open circuit potential directly in the rolling slit of the rolling mill during the cold-rolling information on the surface in the course of the friction can be obtained. The potential can shift into either negative (due to the destruction of the surface oxide layer) or positive (due to the subsequent repassivation of the surface) direction depending on the processes taking place at the surface.

The change in open circuit potential during cold-rolling is a complex synergy of tribochemical, electrochemical and mechanical properties of the rolling mill system. An equilibrium potential of a metal under mechanical stress is different from that of the unstressed metal because of the changes in the free energy. Additionally, different mechanical loading of the electrodes (roller or product) leads to a mixed potential between the loaded and unloaded areas. The speed of the cold rolling and the mechanical stress during rolling affect the electrode potential of the system. Still, a mass transport of the charge carriers inside the electrolyte to and from the electrode plays a great role in open circuit potential since it affects the composition of the double layer near the electrode surface. A stationary situation is established between the destruction and repassivation of the surface structures arises during the continuous cold rolling process. The electrode potential in this progressing state depends on the mode of friction. Under severe conditions leading to the cyclic destruction of the surface and catastrophic wear, followed by its restoration, sharp oscillations of the electrode potential were observed.

The destruction of the oxide layers and exposure of the fresh metallic surface to the aqueous emulsion is accompanied with intensive hydrogen evolution. Atomic hydrogen is adsorbed on the surface and exhibits a pressure supported absorption into the rolls during their whole lifetime in the rolling mill. Galvanic coupling of the working rolls with the product intensifies the hydrogen embrittlement related problems of the rolls. Brittle mechanic failure and blistering of the forged rolls are the consequences of the hydrogen-related wear.

\section{Acknowledgements}

The financial support of this work provided by the German Federal Ministry of Economics and Technology under grant number KF 2001501SU8 is gratefully acknowledged.

\section{References}

[1] I.M. Bernstein, Hydrogen Effects in Materials, in: A.W. Thompson, N.R. Moody (Eds.), TMS, Warrendale, PA, 1996, p. 3

[2] M. Nagumo, ISIJ Int. 41 (2001) 590-598

[3] J.P. Hirth, Metall. Trans. A 11 (1980) 861-890

[4] P. Sofronis, I.M. Robertson, AIP Conference Proceedings 837 (2006) 64-70

[5] S.V. Merzlikin, A.W. Hassel, K. Steinhoff, M. Wildau, Practical Metallography 07 (2011) 365-375

[6] A.W. Hassel, M.M. Lohrengel, Electrochim. Acta 40 (1995) 433-437

[7] G.P. Shpenkov, in Tribology Series. 29 D. Dowson (Ed.), Friction Surface Phenomena, Elsevier, 1995

[8] E. Akiyama, M. Stratmann, A.W. Hassel, J. Phys. D 39 (2006) 3157-3164

[9] E. Abelev, A.J. Smith, A.W. Hassel, Y. Ein Eli, J. Electrochem. Soc. 153 (2006) B337-B343

[10] A.W. Hassel, A.J. Smith, Corros. Sci. 49 (2007) 231-239

[11] D. Weisz-Patrault, A. Ehrlacher, N. Legrand, J. Mater. Process. Technol. 211 (2011) 1500-1509 
[12] A.W. Hassel, K. Fushimi, M. Seo, Electrochem. Commun. 1 (1999) 180-183

[13] J.-P. Celis, P. Ponthiaux, F. Wenger, Wear 261 (2006) 939-946

[14] S. Mischler, S. Debaud, D. Landolt, J. Electrochem. Soc. 145 (1998) 750-758

[15] S. Rossi, F. Deflorian, M. Zen, L. Fedrizzi, Mater. Corros. 51 (2000) 552-556

[16] V.V. Shyrokov, Kh.B. Vasyliv, Mater. Sci. 44 (2008) 646-652

[17] G. Milazzo, S. Caroli, V.K. Sharma, Tables of Standard Electrode Potentials, Wiley, Chichester, 1978

[18] A.J. Bard, R. Parsons, J. Jordan, Standard Potentials in Aqueous Solutions, Marcel Dekker, New York, 1985

[19] S.G. Bratsch, J. Phys. Chem. Ref. Data 18 (1989) $1-21$

[20] D. Landolt, S. Mischler, M. Stemp, S. Barril, Wear 256 (2004) 517-524

[21] D. Landolt, S. Mischler, M. Stemp, Electrochim. Acta 46 (2001) 3913-3929

[22] R. Oltra, B. Chapey, L. Renaud, Wear 186-187 (1995) 533-541

[23] M. Pourbaix, Atlas of Electrochemical Equilibria in Aqueous Solutions, Pergamon Press, Oxford, 1966

[24] V.P. Sholud'ko, Fiz.-Khim. Mekh. Mater. 18 (1982) 89-92
[25] Dedy Ng, Tapajyoti Sen, Feng Gao, Hong Liang, J. Electrochem. Soc. 155 (2008) H520$\mathrm{H} 524$

[26] T.N. Anderson, J.L. Anderson, H. Eyring, J. Phys. Chem. 73 (1969) 3562-3570

[27] G.T. Burstein, M.A. Kearns, J. Electrochem. Soc. 131 (1984) 991

[28] T. Tsuru, Mater. Sci. Eng. A 146 (1991) 1-14

[29] L. Bjornkvist, I. Olefjord, Corros. Sci. 32 (1991) 231-242

[30] D.M. Drazic, J.P. Popic, Corrosion 60 (2003) 297-303

[31] S. Trasatti, J. Electroanal. Chem. 33 (1971) 351378

[32] J. Simao, D.K. Aspinwall, J. Mater. Process. Technol. 92-93 (1999) 281-287

[33] L. Fedrizzi, S. Rossi, F. Bellei, F. Deflorian, Wear 253 (2002) 1173-1181

[34] FARADAYIC ${ }^{\circledR}$ Process for Chromium Plating from a Trivalent Bath, http:// www.faradaytechnology.com/PDFfiles/ IndustrialCoatings

[35] EPA, Final Report: A Cost-Competitive Functional Trivalent Chromium Plating Process To Replace Hexavalent Chromium Plating http://cfpub.epa.gov

[36] J.W. Signorelli, M.J. Serenelli, M.A. Bertinetti, J. Mater. Process. Technol. 211 (2011) 1500-1509 